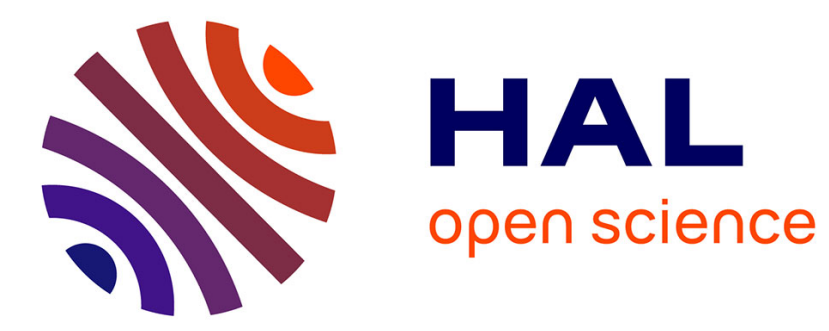

\title{
Dual fluorescence in strap ESIPT systems: a theoretical study
}

Amara Chrayteh, Chris Ewels, Denis Jacquemin

\section{To cite this version:}

Amara Chrayteh, Chris Ewels, Denis Jacquemin. Dual fluorescence in strap ESIPT systems: a theoretical study. Physical Chemistry Chemical Physics, 2020, 22 (2), pp.854-863. 10.1039/C9CP06261K . hal-02553682

\section{HAL Id: hal-02553682 \\ https://hal.science/hal-02553682}

Submitted on 30 Apr 2020

HAL is a multi-disciplinary open access archive for the deposit and dissemination of scientific research documents, whether they are published or not. The documents may come from teaching and research institutions in France or abroad, or from public or private research centers.
L'archive ouverte pluridisciplinaire HAL, est destinée au dépôt et à la diffusion de documents scientifiques de niveau recherche, publiés ou non, émanant des établissements d'enseignement et de recherche français ou étrangers, des laboratoires publics ou privés. 


\title{
Dual fluorescence in strap ESIPT systems: a theoretical study $\dagger$
}

\author{
Amara Chrayteh, ${ }^{\mathrm{ab}}$ Chris Ewels $\quad \star^{\mathrm{b}}$ and Denis Jacquemin $\quad \star^{\mathrm{a}}$
}

Alkylamine-strapped chromophores based on a dithienylpyrrole core, and in which the Excited State Intramolecular Proton Transfer (ESIPT) process yields a zwitterionic structure rather than a keto tautomer have been reported recently (Suzuki et al., Angew. Chem. Int. Ed., 2014, 53, 8231), and are known to exhibit large Stokes shifts. Using Time-Dependent Density Functional Theory (TD-DFT) we investigate the ESIPT mechanism in this family of chromophores considering various substituents and two solvents (cyclohexane and acetone). In order to model the solvent effects, three polarisation models have been applied: the linear response (LR), the corrected linear-response (cLR), and the combination of these two formalisms ( $L R+C L R$ ). The selected protocol is shown to be effective for a series of compounds with known experimental behaviors, and is then applied to novel derivatives with various donor and acceptor groups and heteroatoms. We determine the absorption and emission wavelengths as well as the energies of the different states that play a role in the ESIPT process. We show that the introduction of electron-withdrawing and electron-donating groups plays an important role in achieving redshifted emission from the ESIPT state.

\section{Introduction}

Excited state intramolecular proton transfer (ESIPT) is a fast photo-tautomerization process occurring in the electronically excited state (ES) of molecules possessing a strong intramolecular hydrogen bond (H-bond). ${ }^{1,2}$ ESIPT produces a tautomer presenting an entirely different electronic structure from the initial Franck-Condon form. Consequently, the emission from the ESIPT structure is strongly redshifted as compared to the absorption, and a large Stokes shift is observed. Additionally, dual fluorescence originating from both the canonical and the ESIPT structures can also take place when the two tautomers have

\footnotetext{
${ }^{a}$ Laboratoire CEISAM - UMR CNRS 6230, Université de Nantes,

2 Rue de la Houssinière, BP 92208, 44322 Nantes Cedex 3, France.

E-mail: Denis.Jacquemin@univ-nantes.fr; Tel: +33-2-51-12-55-64

${ }^{b}$ Institut des Matériaux Jean Rouxel (IMN), Université de Nantes, CNRS,

2 rue de la Houssinière, BP 32229, 44322 Nantes cedex 3, France.

E-mail: Chris.Ewels@cnrs-imn.fr; Tel: +33-2-40-37-64-07

$\dagger$ Electronic supplementary information (ESI) available: (i) Comparison between experimental and theoretical fluorescence wavelengths; (ii) $\mathrm{LE} / \mathrm{Z}$ fluorescence wavelengths for compounds 1-4 in acetone; (iii) difference in LE fluorescence wavelengths for compounds 1-5a; (iv) ES dipole moments; (v) CC2 vertical excitation energies; (vi) vertical excitation energies and fluorescence maximum wavelengths computed with TD-DFT compared with the experimental values for 1-5; (vii) distance between the two nitrogen atoms in the ground state; (viii) potential energy diagram for 3 and 4; (ix) distance between the two nitrogen atoms and partial atomic charges in the LE state; (x) HOMO/LUMO plots of 1, 2, and 4; (xi) HOMO/LUMO and EDD plots of 10 and 14; (xii) relative LE/Z energies for substituted compounds 6-17. See DOI: $10.1039 / \mathrm{c} 9 \mathrm{cp} 06261 \mathrm{k}$
}

comparable ES energies. The emission of long-wavelength photons paves the way to many applications including white light-emitting materials, ${ }^{3-5}$ polymorph-dependent light-emitting materials, ${ }^{6}$ and biological fluorescent tags to study DNA-protein interaction, ${ }^{7}$ which explains the growing popularity of ESIPT dyes. Most ESIPT processes take place within molecules containing a five or six-member aromatic ring presenting a strong intramolecular H-bond between the proton donor and acceptor groups. In the majority of the previously studied ESIPT molecules, an amino $\left(\mathrm{NH}_{2}\right)$ or an alcohol $(\mathrm{OH})$ group acts as the proton donor, and a carbonyl group $(-\mathrm{C}=\mathrm{O})$ or an heterocyclic nitrogen atom serves as the proton acceptor. Therefore, the ESIPT reported in most compounds is based on a keto-enol or an amino-imino tautomerization (Fig. 1a). ${ }^{8-11}$ The ground state (GS) of these chromophores typically exhibits the enol form, whereas the keto tautomer is formed through an intramolecular proton transfer along the H-bond between the two $\pi$-conjugated moieties. However, a few exceptions to such ESIPT structures have been reported, such as 2-(hydroxyphenyl)imidazo[1,2-a]pyridines (HPIPs), in which the ESIPT process yields a formal zwitterionic structure rather than a keto isomer (Fig. 1b). ${ }^{12-16}$

A series of new ESIPT chromophores involving a tertiary amine moiety placed in a strap as the proton acceptor and a $\pi$-conjugated pyrrole scaffold as the proton donor was recently designed (Fig. 1c). ${ }^{17}$ The first member of this new class of ESIPT dyes was reported by Suzuki et al. in 2014: they prepared a dye presenting a dithienylpyrrole skeleton capped by dimesitylboryl groups, 2 in Fig. $2{ }^{17}$ This dye may show two emission bands 

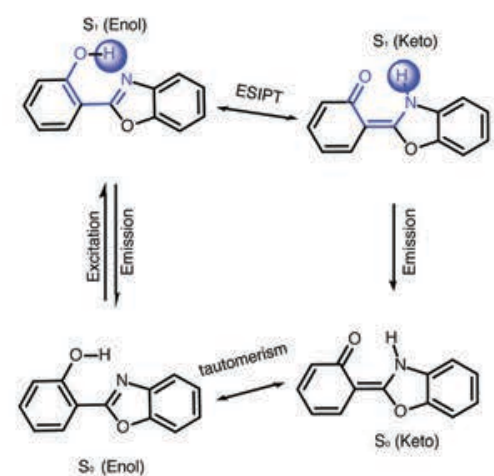

S. (Enol)

$\mid \begin{gathered}\frac{5}{5} \\ \frac{\text { m }}{5} \\ \text { w }\end{gathered}$
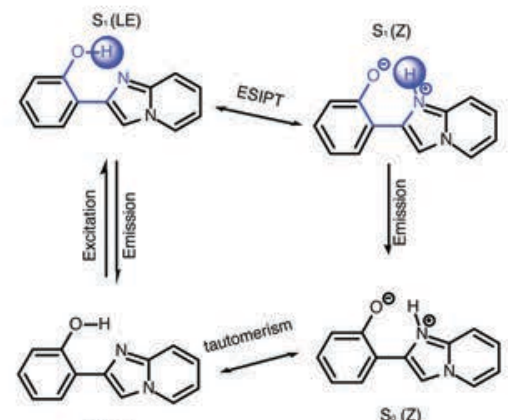

S. (LE)

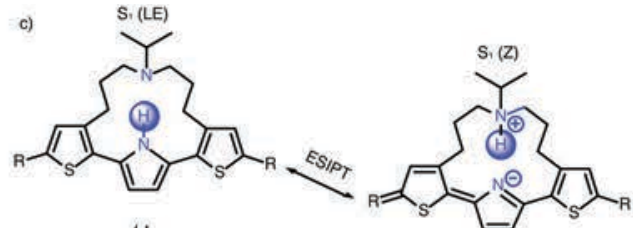

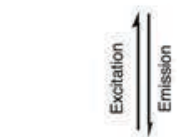

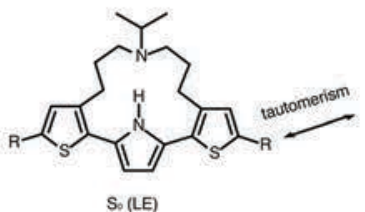

So (LE)

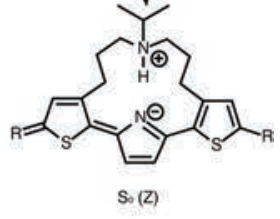

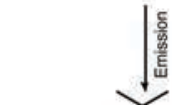

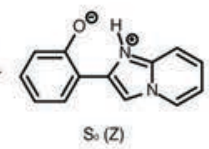

Fig. 1 Representation of the ESIPT process in (a) a benzofuran dye based on the keto-enol tautomerism, (b) an HPIP derivatives that involves zwitterion form, and (c) the systems considered in this work.
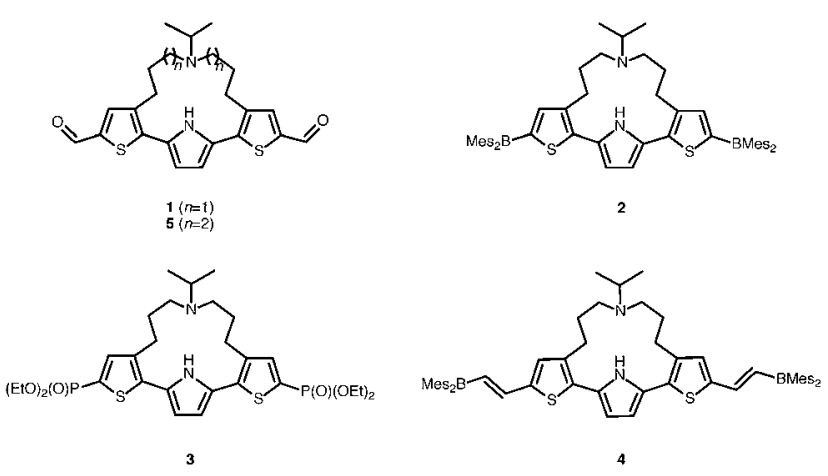

Fig. 2 Molecular structure of the emissive strapped ESIPT compounds experimentally studied in ref. 17 and 21 .

originating from the local excited state (LE) and the zwitterionic (Z) tautomeric forms, the latter being generated by ESIPT. The measured fluorescence wavelengths are strongly dependent on solvent polarity. In cyclohexane, a non-polar solvent, 2 shows a single component emission ( $\lambda_{\text {fluo }}=500 \mathrm{~nm}$ ) attributed to the $\mathrm{LE}$ form, whereas increasing the polarity of the solvent by using acetone leads to a strong bathochromic $\operatorname{shift}\left(\lambda_{\text {fluo }}=602 \mathrm{~nm}\right)$ of the emission, attributed to ESIPT $(\mathrm{Z})$ fluorescence. In order to investigate this phenomenon theoretically, Suzuki et al. performed quantum-chemical calculations for 2 in both gas phase and acetone. ${ }^{17}$ They employed a Time-Dependent DFT (TD-DFT) approach combined with both the CAM-B3LYP ${ }^{18}$ and $\mathrm{PBE0}^{19}$ exchange-correlation functionals, and accounted for the environmental effects using the Solvent Model Density (SMD) method..$^{20} \mathrm{~A}$ comparative analysis showed that the calculated emission wavelength for the ESIPT state was in good agreement with the experimental value, whereas, the computed $\lambda_{\text {fluo }}$ of the LE isomer was redshifted as compared to the measurement. In 2018, the same group proposed a more extensive study evaluating the impact of the substituents at the terminal positions and the influence of the strap length on the optical properties. ${ }^{21}$ This second investigation demonstrated that both the nature of the substituents and the strap length significantly affect the fluorescence spectra and the ESIPT efficiency. In more detail, they tested six compounds, with different substitutions at the terminal position, including compounds 1,3 , and 4 , and one compound with a longer strap 5 (see Fig. 2). They found that the $\mathrm{Z}$ emission depends on the degree of the electron-withdrawing strength of the side group, as this strength affects the acidity of the pyrrole group in the ES. Likewise the chain length of the strap moiety is critical for the formation of the intramolecular $\mathrm{H}$-bond, a too long strap preventing the ESIPT. A compact theoretical study of solvent effects on the LE and $\mathrm{Z}$ forms was also included in ref. 21 using the Polarizable Continuum Model (PCM). ${ }^{22}$ It was found that the energies of both isomers decrease in a polar medium and, more importantly, that the $\mathrm{Z}$ tautomer becomes more stable in the ES when increasing the solvent polarity. Recently, Zhou and Zhao proposed a theoretical study further discussing the ESIPT reaction of 2 in three different solvents. ${ }^{23}$ They analyzed the nature of LE and $\mathrm{Z}$ tautomers along with locating the ESIPT transition state using TD-DFT as well as the second-order Algebraic-Diagrammatic Construction approach $[\operatorname{ADC}(2)] .^{23}$ They found that, in non-polar solvent such as cyclohexane, the energy barrier is high and thus, fluorescence originates mainly from the LE structure. In contrast, an increase in solvent polarity decreases the energy barrier for the ESIPT reaction and the fluorescence should mainly result from the $\mathrm{Z}$ form. To the best of our knowledge there are no other theoretical nor experimental studies on these systems.

The wide range of applicability of molecules exhibiting redshifted emission motivated us to use advanced theoretical tools to design novel ESIPT systems based on the strap scaffold. To begin with, we consider all previously reported compounds, $\mathbf{1 - 5},{ }^{17,21}$ displayed in Fig. 2 to study their fluorescence properties using a coherent theoretical model. We aim to: (i) determine if the selected protocol can reproduce the experimental outcomes; (ii) provide theoretical insight into their working mechanism; (iii) investigate unknown derivatives in an effort to design dyes with new spectroscopic signatures. For that reason, we choose the M06- $2 \mathrm{X}^{24}$ hybrid exchange-correlation functional in the present 


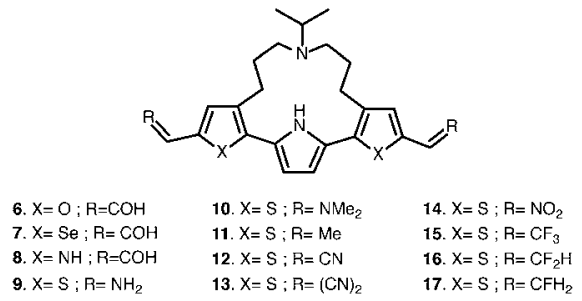

Fig. 3 Molecular structure of the novel derivatives studied here.

work, since it has been reported to provide accurate transition energies for a large panel of ES and is also suited for various classes of ESIPT dyes. ${ }^{25-31}$ Thereafter, the effect of substituents is evaluated by modifying the terminal functional group and the heteroatom of the side rings in 1, resulting in the design of twelve new derivatives 6-17 (Fig. 3). In addition the solvatochromic effects are analyzed for these molecules by applying various solvent models including both linear response (LR) and state specific (SS) schemes to offer a higher accuracy in the modeling of the environment. The ESIPT mechanism is explored as well by locating the transition state (TS) connecting the LE and $\mathrm{Z}$ structures in the ES.

\section{Computational details}

Density Functional Theory (DFT) and Time-Dependent DFT (TD-DFT) calculations are carried out using the Gaussian16 package. $^{32}$ The impact of the solvent, cyclohexane $(\varepsilon=2.02)$ or acetone $(\varepsilon=20.7)$, is accounted for using the integral equation formalism (IEF) version of the PCM approach. For all compounds, the GS and the ES geometries are fully optimized using DFT and TD-DFT respectively, combined with the M06- $2 \mathrm{X}^{24}$ hybrid exchange-correlation functional. The $6-31+G(d, p)$ atomic basis set is used during the geometry optimisations, whereas a larger atomic basis set, $6-311+\mathrm{G}(2 \mathrm{~d}, \mathrm{p})$, is selected to compute the total and transition energies on these optimal geometries using the same functional. Next, vibrational frequency calculations are performed for both states using the optimized geometries to verify that all structures are true minima of the potential energy surface, as well as to obtain the zero-point vibrational energies (ZPVE) for both states. Consistently, the ESIPT transition state geometries are optimized at the TD-DFT level using M06-2X functional and the $6-31+\mathrm{G}(\mathrm{d}, \mathrm{p})$ atomic basis set. These TS present a single imaginary frequency corresponding to the proton transfer process. Since we focus on excited-state calculations with PCM, and in order to obtain the best theoretical estimates of solvent effects, three different models are used in this work for computing the solvent impact on the energies: (i) the Linear Response approach $^{33,34}$ which is a method well-suited for local bright ES that are characterized by a small change in the electronic density; such model is however less effective for charge-transfer excitations; (ii) the corrected LR (cLR) model that accounts for SS effects in a single-shot perturbative way; cLR is well suited when the transition involves a large density reorganization; ${ }^{35}$ and (iii) the combination of these two formalisms together
$(\mathrm{LR}+\mathrm{cLR})^{36-39}$ which is more general and adequate for, e.g., states that are both bright and involving significant changes of the electronic density. Indeed, the LR + cLR model recovers all major solvation effects and often yields more accurate total and transition energies than the LR and cLR approaches taken separately. Since geometrical relaxation is a slow process, excited-state geometries have been determined in the equilibrium limit of the LR-PCM approach. Thus, vibrational frequencies, including zero point corrections and 0-0 energies, are considered in this limit as well. In contrast, both the absorption and emission energies are given in the nonequilibrium PCM limit, except for LR-PCM fluorescence energies, that are in equilibrium due to the intrinsic limit of the current implementations of this model. In addition, to ascertain for one case our TD-DFT results, we use the second-order Coupled Cluster model, CC2, with the aug-cc-pVDZ basis set. CC2 calculations are done with the Turbomole package ${ }^{40}$ applying the RI approach. These CC2 calculations are performed in gas phase and the solvent effects, as computed with TD-DFT, are next added to estimate the CC2 results in solution, following a previously described protocol. ${ }^{29}$ We note that condensed phase CC calculations are also possible, ${ }^{41,42}$ but such calculations have not been carried out here.

\section{Results and discussion}

\subsection{Impact of the solvent model on fluorescence wavelengths}

In this section, we perform a comparative analysis of theoretical and experimental fluorescence wavelengths. We compute the emission energies of molecules 1-5 displayed in Fig. 2 in two different solvents (cyclohexane and acetone). Note that compound 5 presents two conformers (5a and $\mathbf{5 b}$, see below), and, we consider the most stable one in the statistics. To determine which solvent model could be viewed as the most appropriate, three PCM models are evaluated (LR, cLR, and LR + cLR). At this stage, it should be reminded that the vertical fluorescence energies are not strictly equivalent to measured wavelengths of maximal emission. ${ }^{43}$ However, given the size of the systems, one has to resort to this vertical approximation which is reasonable in a homologous series of compounds. Three different criteria are taken into account to appraise the solvent models: (i) the difference between experimental and theoretical absolute fluorescence wavelengths computed for the two tautomers (LE and $\mathrm{Z}$ ), (ii) the difference between fluorescence wavelengths originating from $\mathrm{LE}$ and $\mathrm{Z}$ in acetone, and (iii) the difference between LE emission wavelengths when going from one solvent to another. Our data are collected in Tables S1-S3 in the ESI. $\dagger$ For the first criterion, it can be seen that globally and, especially for the emission originating from the $\mathrm{Z}$ tautomer, $\mathrm{LR}+\mathrm{cLR}$ gives the smallest deviations between experiment and theory. Table 1 provides a statistical analysis confirming this statement. Indeed, it can be noted that even if cLR gives the smallest MSE $-0.056 \mathrm{eV} /-0.054 \mathrm{eV}$ and MAE $0.080 \mathrm{eV} / 0.098 \mathrm{eV}$ for the $\mathrm{LE}$ fluorescence in cyclohexane/ acetone, $\mathrm{LR}+\mathrm{cLR}$ provides tiny $\operatorname{MSE}(-0.020 \mathrm{eV})$ and MAE 
Table 1 Statistical data obtained from the comparison of experimental and theoretical emission energies (Table S1 in the ESI): mean signed error (MSE) and mean absolute error (MAE). All values are given in $\mathrm{eV}$

\begin{tabular}{lllrl}
\hline Solvent & State & Method & \multicolumn{1}{c}{ MSE } & MAE \\
\hline Cyclohexane & LE & LR & 0.038 & 0.102 \\
& & cLR & -0.056 & 0.080 \\
Acetone & LE & LR + cLR & 0.050 & 0.098 \\
& & LR & 0.140 & 0.164 \\
& cLR & -0.054 & 0.098 \\
& Z & LR + cLR & 0.198 & 0.198 \\
& & LR & -0.107 & 0.107 \\
& & LR & -0.262 & 0.262 \\
& All & LRR & -0.020 & 0.050 \\
& & cLR & & 0.125 \\
& & LR + cLR & & 0.138 \\
& & &
\end{tabular}

$(0.050 \mathrm{eV})$ for the $\mathrm{Z}$ emission as well as the overall smallest MAE $(0.120 \mathrm{eV})$. For the second criterion, we notice from Table S2 in the ESI $\dagger$ that the computed difference between LE and $\mathrm{Z}$ emission wavelengths are systematically too small regardless of the selected solvent model. It is nevertheless clear that LR + cLR gives slightly better correlation with experiment as compared to LR and cLR taken separately, e.g. a theoretical difference of $-56 \mathrm{~nm}$ is found between the LE and $\mathrm{Z}$ fluorescence wavelengths for compound 2 in acetone with LR + cLR, whereas the experimental data provides a difference of $-82 \mathrm{~nm}$. Larger errors are obtained with both LR and cLR $(-41 \mathrm{~nm}$ and $-46 \mathrm{~nm}$ respectively). Similar findings are obtained for the other molecules. Let us now move to the third criterion, the magnitude of the solvatochromism for which we note that cLR yields slightly smaller values than experiment, whereas both LR and LR + cLR tend to overshoot the measured values (Table S3 in the ESI $\dagger$ ). Therefore, these analyses indicate quite obviously that LR is not suitable for the investigated systems, whereas both cLR and LR + cLR appear to be more reasonable which is not a surprising outcome for ESIPT dyes involving both a large reorganization of the electron density between the GS and the ES, and two tautomers of different polarities (see below). Given that LR + cLR has globally the edge, we apply systematically this model in the following.

\subsection{Influence of the solvent polarity}

Our theoretical studies of compound $\mathbf{1}$ in cyclohexane and acetone demonstrate a strong dependency between the relative stabilization of LE and $\mathrm{Z}$ tautomers and the solvent polarity (Fig. 4). As expected, two local minima are obtained in the ES, one associated with LE and another with Z. A single component emission from LE is clearly predicted for $\mathbf{1}$ in cyclohexane as the LE form is more stable than $\mathrm{Z}$ by $0.1 \mathrm{eV}$, so there is no driving force for ESIPT. In contrast, the $\mathrm{Z}$ tautomer is significantly stabilized in acetone and consequently becomes more stable than LE. Provided ESIPT is faster than emission, which is likely given the fact that the experimentally measured fluorescence lifetimes are of the order of the ns, this potential energy surface indicates a possible ESIPT and, hence, a strong bathochromic shift of the emission when going from LE cyclohexane to

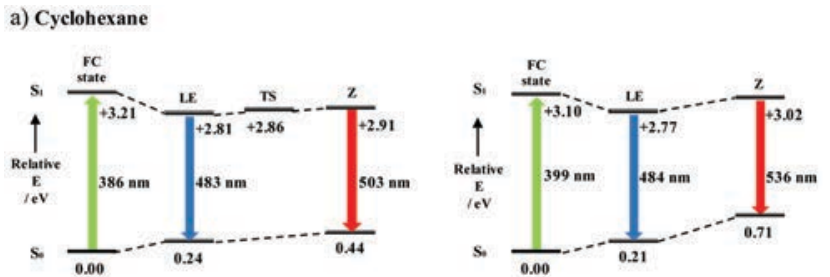

b) Acetone
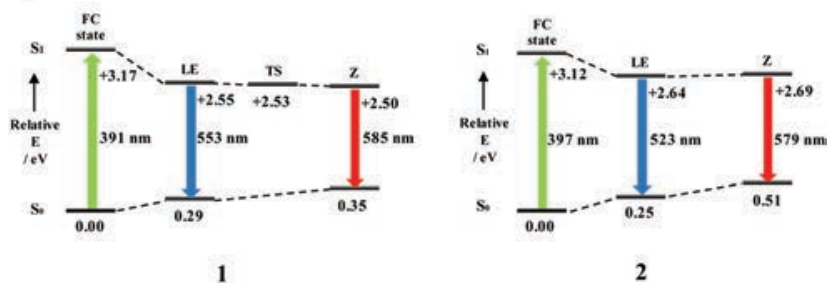

Fig. 4 Potential energy diagrams ( $E+$ ZPVE) for compound $\mathbf{1}$ and $\mathbf{2}$ obtained in (a) cyclohexane and (b) acetone using the LR + cLR solvent model. Theoretical energies and wavelengths have been obtained from TD-DFT calculations using the M06-2X functional and the $6-311+G(2 d, p)$ atomic basis set. For 2, a very large compound, the determination of the TS was technically beyond reach.

$\mathrm{Z}$ in acetone $\left(\Delta \lambda_{\text {fluo }}=102 \mathrm{~nm}\right)$. To understand this large solvent effect, the ES dipole moment of the two tautomers has been determined in both solvents. The dipole moment of the $\mathrm{Z}$ tautomer is much larger than that of its canonical counterpart, and, more importantly, it significantly increases with the increase of solvent polarity: $\mu_{\mathrm{ES}}=10.6 / 13.3 \mathrm{D}$ and $\mu_{\mathrm{ES}}=15.1 / 19.1 \mathrm{D}$ for $\mathrm{LE} / \mathrm{Z}$ in cyclohexane and acetone, respectively (Table S4 in the ESI†). This clearly supports the zwitterionic character of the tautomer after ESIPT and explain its very strong stabilization in acetone. For the records, we have also investigated the possibility to form the anion in the $\mathrm{ES}$ of $\mathbf{1}$, i.e., the $\mathbf{1}^{\mathrm{LE}}+$ acetone $\rightarrow \mathbf{1}^{-}+$acetone $\cdot \mathrm{H}^{+}$. However, this reaction was found to be strongly endergonic $\left(+47 \mathrm{kcal} \mathrm{mol}^{-1}\right)$, and it can be likely be ruled out. This is also consistent with the experimental data of Suzuki and coworkers who reported no significant change of the emission even in water. ${ }^{17}$ To further analyze the ESIPT process, let us investigate the nature of the ES in 1. Fig. 5 shows the frontier molecular orbitals together with the Electron Density Difference (EDD) plots computed in acetone. As expected, the first excited state presents a typical $\pi \rightarrow \pi^{*}$ topology, the changes of densities being localized on the conjugated moiety of the dye. There is a partial quadrupolar like charge-transfer from the central pyrrole towards the external aldehyde acceptors. Although the pyrrole is losing density after absorption, its nitrogen atom seems to undergo a slight $\pi$ density gain in the lowest ES. Nevertheless this donating nitrogen atom becomes slightly less negative in the ES $(q=-0.02 e)$, whereas, the accepting nitrogen atom is highly negative in both states $(q=-0.33 e)$, hence it is "willing" to attract a positive charge, which is favorable for triggering ESIPT.

The theoretical TS for the proton transfer of $\mathbf{1}$ in cyclohexane was found to be more stable than the $\mathrm{Z}$ form by $c a .0 .05 \mathrm{eV}$ after inclusion of the ZPVE correction (there is a small maximum on the total energy potential energy surface). This suggests a barrierless "back-ESIPT" and hence the infeasibility of the 
LUMO

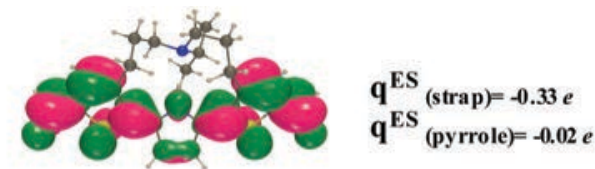

HOMO

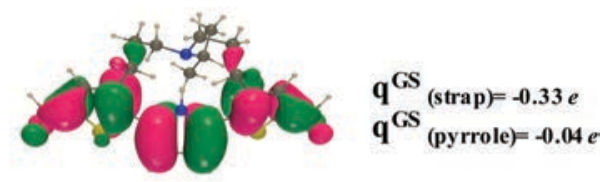

EDD

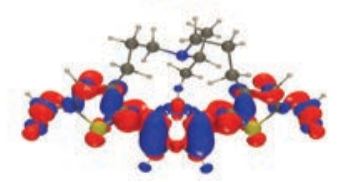

Fig. 5 Representation of the Highest Occupied Molecular Orbitals (HOMO), Lowest Unoccupied Molecular Orbitals (LUMO) and the Electron Density Difference (EDD) plots (isovalue: 0.02 au for MOs 0.0015 au for EDD) of compound 1 in acetone. The red and blue areas in the EDD represent the increase and decrease of electron density upon photon absorption, respectively. We also provide the GS and ES Mulliken charges computed for the two nitrogen atoms.

emission from $\mathrm{Z}$ tautomer in this solvent. In sharp contrast, the forward process is barrierless in acetone, facilitating the formation of $\mathrm{Z}$ tautomer, and consistently only the emission from this structure can be expected to occur according to our calculations (Fig. 4). These findings are in excellent agreement with the experimental observations that show LE emission in cyclohexane but $\mathrm{Z}$ fluorescence in acetone. ${ }^{21}$ In addition, due to the limits of TD-DFT, that has an error bar significantly larger than $0.05 \mathrm{eV},{ }^{26} \mathrm{CC} 2$ calculations were performed for 1 on the geometries obtained from TD-DFT (see Table S5 in the ESI $\dagger$ ). However, the CC2-corrected potential energy surface yields a slightly disfavored ESIPT formation in acetone. Therefore, CC2 corrections do not improve correlation with experiment, ${ }^{21}$ and we therefore stick to TD-DFT in the following.

Similar analyses can also be performed for molecules 2, 3, and 4 (see also Section 3.4). According to our calculations, the fluorescence of $\mathbf{2}$ is originating only from the canonical tautomer in cyclohexane. Indeed, as can be seen in Fig. 4, the LE form is more stable by $c a .0 .25 \mathrm{eV}$ compared to the ESIPT structure. In acetone, the $\mathrm{Z}$ state is significantly stabilized and consequently presents an energy comparable to its LE counterpart, indicating that proton transfer becomes a more accessible process. Nevertheless, we notice that for 2 , the $\mathrm{Z}$ tautomer remains slightly less stable than the LE one, by $0.05 \mathrm{eV}$ in acetone. This is not totally satisfying as Z emission is observed experimentally. This discrepancy is simply due to the limitations of TD-DFT or to the static picture used in this work. We note that a similar problem is also observed in the theoretical calculations performed by Suzuki et al. ${ }^{17}$ Despite this small discrepancy, the global trend nicely fits experiment: ${ }^{17}$ the theoretical emission wavelengths for compound 2 in both cyclohexane $\left(\lambda_{\mathrm{Fluo}}^{\mathrm{LE}}=484 \mathrm{~nm}\right)$ and acetone $\left(\lambda_{\text {Fluo }}^{\mathrm{LE}}=523 \mathrm{~nm} / \lambda_{\text {Fluo }}^{\text {ESIPT }}=579 \mathrm{~nm}\right)$ are in reasonable agreement with the measured values in cyclohexane $\left(\lambda_{\text {Fluo }}^{\mathrm{LE}}=500 \mathrm{~nm}\right)$ and acetone $\left(\lambda_{\text {Fluo }}^{\mathrm{LE}}=520 \mathrm{~nm} / \lambda_{\mathrm{Fluo}}^{\mathrm{ESIPT}}=602 \mathrm{~nm}\right),{ }^{17}$ see Table $\mathrm{S} 6$ in the ESI. $\uparrow$ Thus, our theoretical $\mathrm{LE} / \mathrm{Z}$ wavelength difference correlates reasonably with experiment. Similar findings are obtained for compound 4, see Fig. S1 and Table S6 in the ESI. $\dagger$

Compound 3 exhibits experimentally a single emission band in cyclohexane at $417 \mathrm{~nm}$ originating from LE, which matches our theoretical calculations ( $\lambda_{\text {Fluo }}^{\mathrm{LE}}=440 \mathrm{~nm}$, see Fig. $\mathrm{S} 1$ in the ESI $\dagger$ ). In acetone, two emission bands are obtained experimentally ${ }^{21}$ at $420 \mathrm{~nm}$ and $490 \mathrm{~nm}$, but it is not easy to attribute them to the $\mathrm{LE}$ or the $\mathrm{Z}$ tautomers. Indeed, according to our calculations, the two isomers should show a very small energy difference (ZPVE-corrected $\Delta E=-0.02 \mathrm{eV}$ ) within the error bar of theory see also (Fig. S1 in ESI $\dagger$ ). In addition, regarding the emission wavelengths, the experimental $\mathrm{LE} / \mathrm{Z}$ differences for $\mathbf{1}, \mathbf{2}$, and $\mathbf{4}$ in acetone are quite large, e.g., $\left(\Delta \lambda_{1}=-78 \mathrm{~nm}, \Delta \lambda_{2}=-82 \mathrm{~nm}\right.$, and $\left.\Delta \lambda_{4}=-123 \mathrm{~nm}\right)$ in reasonable agreement with the TD-DFT results $\left(\Delta \lambda_{1}=-32 \mathrm{~nm}, \Delta \lambda_{2}=-56 \mathrm{~nm}\right.$, and $\left.\Delta \lambda_{4}=-78 \mathrm{~nm}\right)$. In contrast, for 3 we compute a very small emission wavelength difference between these two tautomers $\left(\Delta \lambda_{3}=-15 \mathrm{~nm}\right.$, see Table S6 in the ESI $\dagger$ ) much smaller than the experimental value $(-70 \mathrm{~nm})$. This motivated us to further look at the experimental spectra (Fig. S2 in ref. 21) and, it appeared that the most intense emission peak appears at $c a .445 \mathrm{~nm}$ (apparently not around $417 \mathrm{~nm}$ ) in cyclohexane which is also close to the acetone " $\mathrm{Z}$ band" that seems to appear at $460 \mathrm{~nm}$ (and apparently not around $490 \mathrm{~nm}$ ) experimentally. Note that such $\Delta \lambda_{3}$ of $-15 \mathrm{~nm}$ seems to more reasonably fit our predictions. In general the experimental fluorescence spectrum of $\mathbf{3}$, as displayed in the original paper, bears little relation with those of the other dyes for which ESIPT is very clear. Consequently, we cannot make a definitive conclusion regarding the origin of the emission of 3 , and the actual existence of ESIPT or not in that compound would likely deserve more experimental investigations.

\subsection{Impact of the strap chain length on ESIPT}

The effect of the strap chain length on the ESIPT behavior was studied experimentally by comparing compounds $\mathbf{1}$ and $\mathbf{5}$ (see Fig. 2). ${ }^{21}$ In our calculations, two initial geometries have been considered for the optimization of $\mathbf{5}$, i.e., the crystallographic structure $\mathbf{5 a}$ and a chemically-intuitive structure $\mathbf{5 b}$ in which the two nitrogen atoms are closer (Fig. 6). The conformer corresponding to the $\mathrm{X}$-ray like structure, $\mathbf{5 a}$, is slightly more stable than the more compact $5 \mathbf{b}$, by $3.74 \mathrm{kcal} \mathrm{mol}^{-1}$ and $4.50 \mathrm{kcal} \mathrm{mol}^{-1}$ in cyclohexane and acetone, respectively. Interestingly geometric and energy differences are also observed

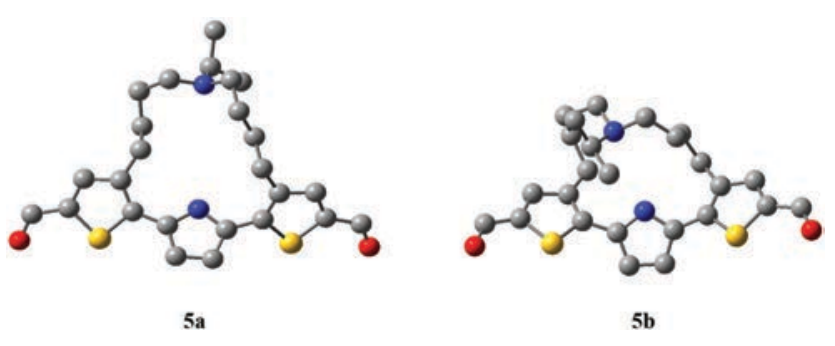

Fig. 6 Representation of the two different optimized ground-state structures of $\mathbf{5}$ in cyclohexane: the X-ray-like structure (5a) and a new conformer obtained by molecular modeling $(\mathbf{5 b})$, both being optimized using the M06-2X functional and the $6-31+G(d, p)$ atomic basis set. Hydrogen atoms omitted for clarity. 
in the ES. For 5a, the LE state is more stable than the ESIPT form (in both solvents), indicating that the emission in 5a occurs from LE only, which fits the experimental data ${ }^{21}$ (Table S6 in ESI $\dagger$ ). The large distance between the proton donor and acceptor (4.22 $\AA$ ) makes the identification of the ESIPT TS of 5a effectively impossible. Nevertheless, the stretching of the $\mathrm{N}-\mathrm{H}$ bond by $0.40 \AA$ results into a dramatic increase in the ES energy (+1.5 eV), hinting that the TS is even higher, confirming that ESIPT is impossible in such a conformation. The LE structure of $\mathbf{5 b}$ remains more stable than the $\mathrm{Z}$ tautomer in cyclohexane, whereas the $\mathrm{Z}$ structure of $\mathbf{5 b}$ becomes the most stable in acetone, similar to the case of compound 1, causing a bathochromic shift of the emission band. Additionally, the ESIPT barrier in $\mathbf{5 b}$ is quite small ( $c a .0 .09 \mathrm{eV}$ ) indicating that the transfer process is energetically accessible with such conformation. Therefore, theory shows that there can be ESIPT in this compact form, and that the absence of ESIPT experimentally is only due to a GS conformational effect. One can speculate that using another type of long strap, allowing the simultaneous presence of several GS conformers, could lead to ESIPT even if the two nitrogen atoms are distant in some conformations.

\subsection{Influence of terminal substituents}

Compounds 1, 2, and 4 have identical skeleton and strap chain length but differ in their terminal substitutions. In order to rationalize these auxochromic effects on the ESIPT phenomenon, we compare their theoretical LE/Z relative energy difference $\left(\Delta E^{\mathrm{ES}}\right)$ with their experimental relative emission peak intensities in Table 2. If the level of theory used would be perfectly accurate one would expect the energy difference $\Delta E^{\mathrm{ES}}=0.00 \mathrm{eV}$ to be the limit separating dominant $\mathrm{LE}$ and $\mathrm{Z}$ emissions. Indeed, a positive $\Delta E^{\mathrm{ES}}$ indicates that ESIPT is a process favored by the excited-state energy landscape and a strong $\mathrm{Z}$ emission is expected, whereas a significantly negative $\Delta E^{\mathrm{ES}}$ should correspond to cases where a dominant LE emission is obtained experimentally. As can be noted in Table 2, in cyclohexane the LE form is always more stable than the $\mathrm{Z}$ form by at least $0.1 \mathrm{eV}$ for the three compounds. This is perfectly consistent with the experimental observations (100\% LE emission). As explained above, 1 shows a clear change in the relative stabilities of $\mathrm{Z}$ and $\mathrm{LE}$ when going to acetone, hence, the zwitterion becomes favored $\left(\Delta E^{\mathrm{ES}}=0.05 \mathrm{eV}\right)$. Experimentally, the $\mathrm{Z}$ emission is strongly dominant (a relative peak intensity of $98 \%$ of $\mathrm{Z}$ ) and only a trifling emission (2\%) is detected for the LE form of $1 .^{21}$ For the two other compounds (2 and 4), we notice a strong stabilization of $\mathrm{Z}$ when going from cyclohexane to acetone. This stabilization decreases the energy difference between the two species and makes them very close $\left(\Delta E_{2}^{\mathrm{ES}}=\right.$ $-0.05 \mathrm{eV}$ and $\Delta E_{4}^{\mathrm{ES}}=-0.03 \mathrm{eV}$ ). As already discussed, these two compounds exhibit experimentally a strong peak of $\mathrm{Z}$ and a weak LE band. Interestingly, the LE emission of 2 and 4 experimentally show higher relative intensities $7 \%$ and $8 \%$ respectively) than in $\mathbf{1}$. This indicates that the theoretical trends are indeed accurate, although the TD-DFT energies seem to slightly overstabilize LE as compared to $\mathrm{Z}$. Consequently, according to the relative energy values obtained above and the experimental observations, it appears that a pure $\mathrm{Z}$ emission can be obtained for $\Delta E^{\mathrm{ES}} \geq-0.05 \mathrm{eV}$.

Furthermore, we measured the distance between the two nitrogens $(\mathrm{N}-\mathrm{N})$ in the GS for 1, 2, and 4 (Table S7 in the ESI $\dagger$ ). The distance between these two nitrogens atoms becomes shorter when going from cyclohexane to acetone, and more importantly, this distance is found to be significantly shorter in $\mathbf{1}$ than in $\mathbf{2}$ and $\mathbf{4}$. This is consistent with an easier ESIPT in $\mathbf{1 .}$ Our theoretical distances are also very similar to the X-ray values. $^{17,21}$ In addition, the partial atomic charges of these two nitrogens in the ES are shown in Fig. S2 in the ESI. $\dagger$ The charges are very similar in cyclohexane and acetone. In contrast, the charge of the nitrogen of the strap moiety of 1 appears to be more negative than the one of 2 and 4 in both solvents, and thus, has a higher tendency to accept the proton. These findings are consistent with the fact that the proton transfer is easier in $\mathbf{1}$ than in $\mathbf{2}$ and $\mathbf{4}$, confirming that simple qualitative criteria can provide first insights into the ESIPT mechanism.

Let us now turn towards the emission wavelengths of the three compounds (Table 2). The experimental ranking of the redshifted emission originating from the $\mathrm{Z}$ tautomer is $\mathbf{4}>\mathbf{2}>\mathbf{1}$ in acetone. This experimental ranking is rather consistent with the TD-DFT one: $\mathbf{4}>\mathbf{1} \simeq \mathbf{2}$. This ordering can be explained: the electron withdrawing $\mathrm{BMes}_{2}$ groups in $\mathbf{4}$ are located in an extended $\pi$-conjugated moiety, which yields an improved delocalization of the LUMO (see Fig. S3 in the ESI $\dagger$ ). Consequently, 4 exhibits a lower LUMO than that of $\mathbf{1}$ and $\mathbf{2}$ and, hence a more redshifted emission is observed. In contrast, $\mathbf{1}$ and $\mathbf{2}$, that contain formyl and $\mathrm{BMes}_{2}$ groups respectively, show very small emission wavelength difference $(\Delta \lambda=6 \mathrm{~nm})$, indicating that both substituents exert similar effects according to our calculations, consistent with the topologies of the frontier MOs (Fig. S3, ESI $\dagger$ ).

Table 2 Energy difference $\Delta E^{E S}(E+$ ZPVE) between LE and Z tautomers for compounds 1, 2, and 4 in cyclohexane and in acetone together with the computed emission wavelengths for the emissive species in comparison with the experimental values. All the theoretical energies values have been obtained from TD-DFT using the M06-2X functional and the 6-311+G(2d,p) atomic basis set. The LR + CLR PCM model is applied. All the relative LE/Z energies are given in $\mathrm{eV}$, whereas emission wavelengths are given in $\mathrm{nm}$

\begin{tabular}{|c|c|c|c|c|c|c|c|c|}
\hline & \multicolumn{4}{|c|}{ Cyclohexane } & \multicolumn{4}{|l|}{ Acetone } \\
\hline & \multicolumn{2}{|l|}{ Theory } & \multicolumn{2}{|l|}{ Experiment } & \multicolumn{2}{|l|}{ Theory } & \multicolumn{2}{|l|}{ Experiment } \\
\hline & $\Delta E_{\mathrm{LR}+\mathrm{cLR}}^{\mathrm{ES}}$ & $\lambda_{\mathrm{LE}} / \lambda_{\mathrm{ESIPT}}$ & LE/Z peak intensity & $\lambda_{\mathrm{LE}} / \lambda_{\mathrm{ESIPT}}$ & $\Delta E_{\mathrm{LR}+\mathrm{cLR}}^{\mathrm{ES}}$ & $\lambda_{\mathrm{LE}} / \lambda_{\mathrm{ESIPT}}$ & LE/Z peak intensity & $\lambda_{\mathrm{LE}} / \lambda_{\mathrm{ESIPT}}$ \\
\hline 1 & -0.10 & $483 /-$ & $100 \% /-$ & $466 /-$ & 0.05 & $553 / 585$ & $2 \% / 98 \%$ & $490 / 568$ \\
\hline 2 & -0.25 & $484 /-$ & $100 \% /-$ & $500 /-$ & -0.05 & $523 / 579$ & 7\%/93\% & $520 / 602$ \\
\hline 4 & -0.26 & $538 /-$ & $100 \% /-$ & $547 /-$ & -0.03 & $608 / 686$ & $8 \% / 92 \%$ & $585 / 708$ \\
\hline
\end{tabular}


In short, the theoretical emission wavelengths have the expected accuracy for such TD-DFT simulations, while the computed relative ES energies are found to be in slight favor of the LE form.

\subsection{Design of novel derivatives substitution effects}

A series of unknown compounds, 6-17, containing both electron-donating groups (EDG) and electron-withdrawing groups (EWG) of different strengths at the terminal positions (R) and various heteroatoms (X) have been designed (Fig. 3). In order to predict the experimental outcomes, we list in Table 3 the energy difference $(E+$ ZPVE) between the two tautomers in both cyclohexane and acetone, along with the computed emission wavelengths for the emissive species. Dye $\mathbf{1}$ is chosen as the reference compound to understand the impact of substituents on ESIPT as well as to determine what kind of emission will likely appear in the spectrum.

Let us first evaluate the impact of the heteroatom on the relative stabilities of the $\mathrm{LE}$ and $\mathrm{Z}$ forms by replacing the sulfur atom $(\mathrm{X}=\mathrm{S})$ in 1 by $\mathrm{X}=\mathrm{O}, \mathrm{X}=\mathrm{Se}$, and $\mathrm{X}=\mathrm{NH}$ in 6,7 , and 8 respectively. The two former appear to have very similar $\Delta E^{\mathrm{ES}}$ values in both solvents as compared to $\mathbf{1}$. One can therefore predict a LE emission in cyclohexane and a $\mathrm{Z}$ emission in acetone. Interestingly, theory predicts a significant redshift for the emission of the seleno derivative in acetone $(611 \mathrm{~nm})$, see Table 3. In order to understand the reason behind the similar ESIPT behavior despite the use of atoms with different sizes, we show the distance between the two nitrogen atoms $(\mathrm{N}-\mathrm{N})$ in $\mathbf{1}$, 6, and 7 and the charges of these centers in Fig. 7. No significant change is found in neither the $\mathrm{N}-\mathrm{N}$ distance nor their charges, indicating that the atomic radius of the $\mathrm{X}$ atom has negligible effect on the stability of the LE and $\mathrm{Z}$, hence going down in the chalcogen column should only tune the position of the bands without significantly affecting their relative intensities. Furthermore, for $\mathbf{8}$, the LE form is found

Table 3 Energy difference $\Delta E^{\mathrm{ES}}(E+$ ZPVE) in eV between LE and $\mathrm{Z}$ tautomers for the series of novel 6-17 dyes in cyclohexane and acetone together with the computed emission wavelengths in $\mathrm{nm}$ for the emissive species. Theoretical energies values have been obtained from TD-DFT calculations using the M06-2X functional and the $6-311+G(2 d, p)$ atomic basis set. The LR + CLR PCM model has been applied

\begin{tabular}{|c|c|c|c|c|}
\hline & \multicolumn{2}{|c|}{ Cyclohexane } & \multicolumn{2}{|l|}{ Acetone } \\
\hline & $\Delta E_{\mathrm{LR}+\mathrm{cLR}}^{\mathrm{ES}}$ & $\lambda_{\mathrm{LE}} / \lambda_{\mathrm{ESIPT}}$ & $\Delta E_{\mathrm{LR}+\mathrm{cLR}}^{\mathrm{ES}}$ & $\lambda_{\mathrm{LE}} / \lambda_{\mathrm{ESIPT}}$ \\
\hline 1 & -0.10 & $483 /-$ & 0.05 & $-/ 568$ \\
\hline 6 & -0.13 & $449 /-$ & 0.05 & $-/ 522$ \\
\hline 7 & -0.12 & $501 /-$ & 0.04 & $-/ 611$ \\
\hline 8 & -0.16 & $428 /-$ & -0.03 & $-/ 492$ \\
\hline 9 & $a$ & $444 /-$ & -0.27 & $476 /-$ \\
\hline 10 & $a$ & $456 /-$ & -0.28 & $488 /-$ \\
\hline 11 & $a$ & $427 /-$ & -0.18 & $455 /-$ \\
\hline 12 & -0.10 & $456 /-$ & 0.03 & $-/ 525$ \\
\hline 13 & -0.04 & $585 / 697$ & 0.12 & $-/ 749$ \\
\hline 14 & -0.01 & $514 / 630$ & 0.14 & $-/ 687$ \\
\hline 15 & -0.14 & $427 /-$ & -0.02 & $-/ 472$ \\
\hline 16 & -0.18 & $428 /-$ & -0.06 & $459 /-$ \\
\hline 17 & -0.19 & $432 /-$ & -0.08 & $464 /-$ \\
\hline
\end{tabular}

${ }^{a}$ Optimization of $\mathrm{Z}$ yields directly to the LE form. to be largely stabilized in cyclohexane, and hence, a pure LE emission is expected to be observed. In contrast, in acetone the $\mathrm{LE}$ and $\mathrm{Z}$ tautomers of $\mathbf{8}$ show comparable energies $\left(\Delta E_{8}^{\mathrm{ES}}=\right.$ $-0.03 \mathrm{eV}$ ), so that, according to the reference energetic window defined previously, the proton transfer in $\mathbf{8}$ should remain almost quantitative. Note that $\mathbf{8}$ displays a strongly blueshifted band as compared to 1 .

Let us now investigate the effect of auxochromic groups (a set of both EDG and EWG of different strengths and natures is selected) at $\mathrm{R}$ position. In cyclohexane, only a pure $\mathrm{LE}$ emission is expected to be observed experimentally in almost all cases $\left(\Delta E^{\mathrm{ES}}<-0.05\right.$, see Table 3$)$. The TS for the ESIPT process in these dyes have also been computed and the values are listed in Table S8 in the ESI. $\dagger$ The TS is never indicating a large barrier for the forward ESIPT in cyclohexane, so it should limit the speed of the process. However, there is a clear change in the energy difference between LE and $\mathrm{Z}$ in both 13 and 14 $\left(\Delta E_{13}^{\mathrm{ES}}=-0.04\right.$ and $\Delta E_{14}^{\mathrm{ES}}=-0.01 \mathrm{eV}$ respectively) together with a strong redshift of the LE emission of these two compounds (585 $\mathrm{nm}$ and $514 \mathrm{~nm}$ respectively), hinting that dual emission is likely to occur even in a non-polar solvent for these two dyes. It is worth noting that $\mathbf{1 3}$ and $\mathbf{1 4}$ show the strongest EWG that allow to stabilize the negative charge appearing in the $\mathrm{Z}$ form. In acetone, the insertion of mesomeric EWG (12, 13, and 14) further stabilizes the $\mathrm{Z}$ tautomer making the emission from the latter dominant as in 1. Interestingly, a large bathochromic shift of the emission is predicted by theory for the two latter (749 $\mathrm{nm}$ and $687 \mathrm{~nm}$ for 13 and 14 respectively). The HOMO/ LUMO and EDD plots of $\mathbf{1 4}$ can be found in Fig. S4 (ESI $\dagger$ ). It can be seen that the LUMO of $\mathbf{1 4}$ is very delocalized and that the density on the nitro groups increases upon photon absorption which yields a strong stabilization of the $\mathrm{Z}$ tautomer after ESIPT and hence a large redshift. Adding an inductive EWG (15-17) yields a smaller effect, the $\mathrm{Z}$ tautomer being energetically less favored $\left(\Delta E_{15}^{\mathrm{ES}}=-0.02 \mathrm{eV}, \Delta E_{16}^{\mathrm{ES}}=-0.06 \mathrm{eV}\right.$, and $\left.\Delta E_{17}^{\mathrm{ES}}=-0.08 \mathrm{eV}\right)$, and a unique LE emission in acetone is even predicted for $\mathbf{1 6}$ and 17, contrasting with 1 . For 15 the $\Delta E^{\mathrm{ES}}$ is found to be within the reference energetic window for $\mathrm{Z}$ emission $\left(\Delta E^{\mathrm{ES}}>-0.05 \mathrm{eV}\right)$ and although we predict the proton transfer to be almost quantitative, the emission wavelength predicted for $\mathbf{1 5}$ appears to be strongly blueshifted (472 nm) as compared to 1 . Clearly there is a large amount of possibilities to separately tune the emission wavelengths and the ESIPT process when using EWG.

Let us now turn to EDG. Globally donor groups tend to stabilize the LE form, and consequently tend to yield a sole LE emission. The fact that EDG makes ESIPT more challenging was already reported before in classical enol-keto systems. ${ }^{8} \mathrm{We}$ underline that the impact of the EDG on the relative $\mathrm{LE} / \mathrm{Z}$ energies is much larger than that of EWG, and follows the expected $\mathrm{NMe}_{2}>\mathrm{NH}_{2}>$ Me ranking. When an EDG is inserted $(\mathbf{9}, \mathbf{1 0}$, and 11), the LE tautomer is largely stabilized in acetone $\left(\Delta E_{\mathbf{9}}^{\mathrm{ES}}=-0.27 \mathrm{eV}, \Delta E_{\mathbf{1 0}}^{\mathrm{ES}}=-0.28 \mathrm{eV}, \Delta E_{\mathbf{1 1}}^{\mathrm{ES}}=-0.18 \mathrm{eV}\right)$, and only a single emission originating from this tautomer is expected to be observed, confirming the intuitive prediction. Indeed, adding side donor groups should be detrimental for the formation of the negative charge. In Fig. S4 of the ESI, $\dagger$ we 


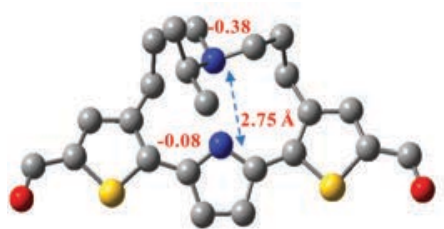

1

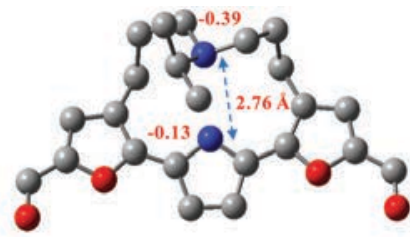

6

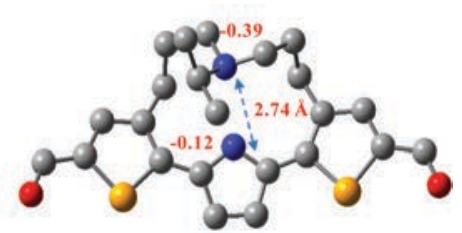

7

Fig. 7 Distance between the two nitrogen atoms and partial Mulliken atomic charge of these atoms in $\AA$ for compounds $\mathbf{1}(X=S), \mathbf{6}(X=O)$ and $7(X=S e)$ in their LE forms in acetone. All values obtained for the ES.

report the HOMO/LUMO and EDD plots of 10: the LUMO of 10 is less delocalized than the HOMO, and the density of $\mathrm{NMe}_{2}$ decreases upon absorption which results in an increase of the basicity of the $\mathrm{N}-\mathrm{H}$ of the pyrrole and tends to inhibit the proton transfer. The computed ESIPT barriers also support this interpretation (Table S8 in the ESI $\dagger$ ).

\section{Conclusions and outlook}

A detailed theoretical study of the ESIPT process occurring in alkylamine-strapped chromophores has been performed with a focus on the impact of chemical substitutions. The ESIPT process in this recently proposed family of dyes yields a zwitterionic structure rather than a keto tautomer. We performed our $a b$ initio calculations by combining TD-DFT method with a continuum solvent approach accounting for both linear-response and state-specific solvent effects (LR + cLR). This model was found more appropriate than the commonly used alternatives, although as all continuum models it does not include the structure of the solvent molecules and hence cannot foresee solvent-assisted proton transfer, which is likely reasonable as the experimental solvents are aprotic. A comparison between the experimental and theoretical values for four compounds 1-4, for which fluorescence spectra have been experimentally measured in both cyclohexane and acetone was performed. According to our calculations, the emission is originating solely from the LE tautomer when cyclohexane is used as a medium. Indeed the energy difference between the two tautomers indicates that ESIPT would yield a less stable tautomer in the ES. When going from cyclohexane to acetone a systematic stabilization of the $\mathrm{Z}$ tautomer compared to LE is obtained, making the emission from the latter (much) more accessible. These conclusions are in good agreement with the experimental observations of Suzuki et al., although the computed relative energies seem to indicate that LE is slightly "overfavored" by TD-DFT. Additionally, theory was able to deliver reasonably accurate fluorescence wavelengths for the four studied compounds.

Several new insights emerge from this current study. First, compound 3 seems to be a less clear-cut case than previously thought. Indeed the experimental fluorescence spectrum as well as the theoretical calculations are likely not clear enough to definitively ascertain the origin of the emissions observed in the polar solvent, and hence the actual existence of ESIPT. This was noticed by the facts that: (i) experimentally, the emission attributed to LE in cyclohexane appears very close to the $\mathrm{Z}$ band in acetone; and (ii) very small energy and wavelength differences are computed between the two tautomers making it challenging to determined whether the emission is resulting from LE or Z. Second, by reexamining compound $\mathbf{5}$ that shows a longer strap, we could locate two different conformers, the most compact being less stable but likely to show ESIPT. This is an example in which exerting pressure on the system could help in developing ESIPT. Third, we proposed and designed 12 new substituted compounds through the introduction of various EWG and EDG at the terminal position ( $\mathrm{R})$ and different heteroatoms (X) (Fig. 3). Since compound 1 shows a clear ESIPT behavior, it was chosen as reference to evaluate the effect of these chemical modifications on both ESIPT and the emission wavelengths. We conclude that (i) replacing sulfur by larger heteroatoms affects only the position of the bands, but not the ESIPT process and can therefore be an interesting approach to "simply" tune the emission spectra; (ii) adding very strong EWG groups, e.g., $\mathrm{NO}_{2}$ or $(\mathrm{CN})_{2}$, should allow dual emission even in cyclohexane, thanks to a strong stabilization of the negative charge present in the $\mathrm{Z}$ tautomer; (iii) using EDG hampers the ESIPT process even in acetone; and (iv) both EDG and EWG strongly modify the emission wavelengths, e.g., we compute an emission at $749 \mathrm{~nm}$ for the $\mathrm{Z}$ form of the compound presenting $(\mathrm{CN})_{2}$ side groups.

In the future it would be very interesting to: (i) investigate how the ESIPT process would evolve in the solid-state, which can now be done by theoretical simulations; ${ }^{44,45}$ (ii) explicitly account for the solvent molecules and their dynamics, as this may significantly impact the transfer process, ${ }^{46}$ especially if these dyes are to be used in a protic environment in which both enhancement and quenching of the ESIPT process have been reported. ${ }^{47-49}$

\section{Conflicts of interest}

There are no conflicts to declare.

\section{Acknowledgements}

The authors thank the RFI LUMOMAT for support in the framework of the Fluo-34 grant. This research used resources of the CCIPL (Centre de Calcul Intensif des Pays de Loire). 


\section{References}

1 J. E. Kwon and S. Y. Park, Adv. Mater., 2011, 23, 3615-3642.

2 F. S. Rodembusch, F. P. Leusin, L. F. Campo and V. Stefani, J. Photochem. Photobiol., A, 2000, 130, 101-111.

3 S. Kim, J. Seo, H. Jung, J.-J. Kim and S. Park, Adv. Mater., 2005, 17, 2077-2082.

4 S. Park, J. E. Kwon, S. H. Kim, J. Seo, K. Chung, S.-Y. Park, D.-J. Jang, B. M. Medina, J. Gierschner and S. Y. Park, J. Am. Chem. Soc., 2009, 131, 14043-14049.

5 K.-C. Tang, M.-J. Chang, T.-Y. Lin, H.-A. Pan, T.-C. Fang, K.-Y. Chen, W.-Y. Hung, Y.-H. Hsu and P.-T. Chou, J. Am. Chem. Soc., 2011, 133, 17738-17745.

6 Y. Sagara, T. Mutai, I. Yoshikawa and K. Araki, J. Am. Chem. Soc., 2007, 129, 1520-1521.

7 J. Zhao, S. Ji, Y. Chen, H. Guo and P. Yang, Phys. Chem. Chem. Phys., 2012, 14, 8803-8817.

8 C. Azarias, S. Budzák, A. D. Laurent, G. Ulrich and D. Jacquemin, Chem. Sci., 2016, 7, 3763-3774.

9 Y. Houari, S. Chibani, D. Jacquemin and A. D. Laurent, J. Phys. Chem. B, 2015, 119, 2180-2192.

10 J. M. Ortiz-Sánchez, R. Gelabert, M. Moreno and J. M. Lluch, ChemPhysChem, 2007, 8, 1199-1206.

11 C.-X. Li, W.-W. Guo, B.-B. Xie and G. Cui, J. Chem. Phys., 2016, 145, 074308.

12 A. J. Stasyuk, P. J. Cywiński and D. T. Gryko, J. Photochem. Photobiol., C, 2016, 28, 116-137.

13 A. Douhal, F. Amat-Guerri and A. U. Acuna, J. Phys. Chem. A, 1995, 99, 76-80.

14 M. R. di Nunzio, Y. Wang and A. Douhal, J. Phys. Chem. B, 2012, 116, 7522-7530.

15 N. Singla and P. Chowdhury, Chem. Phys. Lett., 2012, 548, 71-79.

16 T. Mutai, H. Sawatani, T. Shida, H. Shono and K. Araki, J. Org. Chem., 2013, 78, 2482-2489.

17 N. Suzuki, A. Fukazawa, K. Nagura, S. Saito, H. KitohNishioka, D. Yokogawa, S. Irle and S. Yamaguchi, Angew. Chem., Int. Ed., 2014, 53, 8231-8235.

18 T. Yanai, D. P. Tew and N. C. Handy, Chem. Phys. Lett., 2004, 393, 51-57.

19 C. Adamo and V. Barone, J. Chem. Phys., 1999, 110, 6158-6170.

20 A. V. Marenich, C. J. Cramer and D. G. Truhlar, J. Phys. Chem. B, 2009, 113, 6378-6396.

21 N. Suzuki, K. Suda, D. Yokogawa, H. Kitoh-Nishioka, S. Irle, A. Ando, L. M. G. Abegão, K. Kamada, A. Fukazawa and S. Yamaguchi, Chem. Sci., 2018, 9, 2666-2673.

22 J. Tomasi, B. Mennucci and R. Cammi, Chem. Rev., 2005, 105, 2999-3094.

23 P. Zhou and L. Zhao, Int. J. Quantum Chem., 2018, 118, 25618.

24 Y. T. Zhao and D. G. Truhlar, Theor. Chem. Acc., 2008, 120, 215-241.

25 Y. Houari, A. Charaf-Eddin, A. D. Laurent, J. Massue, R. Ziessel, G. Ulrich and D. Jacquemin, Phys. Chem. Chem. Phys., 2014, 16, 1319-1321.

26 A. D. Laurent and D. Jacquemin, Int. J. Quantum Chem., 2013, 113, 2019-2039.
27 Y. Zhao and D. G. Truhlar, Theor. Chem. Acc., 2008, 120, 215-241.

28 R. Li, J. Zheng and D. G. Truhlar, Phys. Chem. Chem. Phys., 2010, 12, 12697-12701.

29 D. Jacquemin, A. Planchat, C. Adamo and B. Mennucci, J. Chem. Theory Comput., 2012, 8, 2359-2372.

30 M. Isegawa, R. Peverati and D. G. Truhlar, J. Chem. Phys., 2012, 137, 244104.

31 S. S. Leang, F. Zahariev and M. S. Gordon, J. Chem. Phys., 2012, 136, 104101.

32 M. J. Frisch, G. W. Trucks, H. B. Schlegel, G. E. Scuseria, M. A. Robb, J. R. Cheeseman, G. Scalmani, V. Barone, G. A. Petersson, H. Nakatsuji, X. Li, M. Caricato, A. V. Marenich, J. Bloino, B. G. Janesko, R. Gomperts, B. Mennucci, H. P. Hratchian, J. V. Ortiz, A. F. Izmaylov, J. L. Sonnenberg, D. Williams-Young, F. Ding, F. Lipparini, F. Egidi, J. Goings, B. Peng, A. Petrone, T. Henderson, D. Ranasinghe, V. G. Zakrzewski, J. Gao, N. Rega, G. Zheng, W. Liang, M. Hada, M. Ehara, K. Toyota, R. Fukuda, J. Hasegawa, M. Ishida, T. Nakajima, Y. Honda, O. Kitao, H. Nakai, T. Vreven, K. Throssell, J. A. Montgomery, Jr., J. E. Peralta, F. Ogliaro, M. J. Bearpark, J. J. Heyd, E. N. Brothers, K. N. Kudin, V. N. Staroverov, T. A. Keith, R. Kobayashi, J. Normand, K. Raghavachari, A. P. Rendell, J. C. Burant, S. S. Iyengar, J. Tomasi, M. Cossi, J. M. Millam, M. Klene, C. Adamo, R. Cammi, J. W. Ochterski, R. L. Martin, K. Morokuma, O. Farkas, J. B. Foresman and D. J. Fox, Gaussian16 Revision A.03, Gaussian Inc., Wallingford CT, 2016.

33 G. Scalmani, M. J. Frisch, B. Mennucci, J. Tomasi, R. Cammi and V. Barone, J. Chem. Phys., 2006, 124, 094107.

34 R. Cammi and B. Mennucci, J. Chem. Phys., 1999, 110, 9877-9886.

35 M. Caricato, B. Mennucci, J. Tomasi, F. Ingrosso, R. Cammi, S. Corni and G. Scalmani, J. Chem. Phys., 2006, 124, 124520.

36 P. M. Vérité, C. A. Guido and D. Jacquemin, Phys. Chem. Chem. Phys., 2019, 21, 2307-2317.

37 C. A. Guido and S. Caprasecca, Int. J. Quantum Chem., 2019, 119, e25711.

38 S. Corni, R. Cammi, B. Mennucci and J. Tomasi, J. Chem. Phys., 2005, 123, 134512.

39 I. Duchemin, C. A. Guido, D. Jacquemin and X. Blase, Chem. Sci., 2018, 9, 4430-4443.

40 TURBOMOLE V6.6 2014, a development of University of Karlsruhe and Forschungszentrum Karlsruhe $\mathrm{GmbH}$, 1989-2007, TURBOMOLE GmbH, since 2007; available from http://www.turbomole.com.

41 M. Caricato, J. Chem. Theory Comput., 2012, 8, 4494-4502.

42 B. Lunkenheimer and A. Köhn, J. Chem. Theory Comput., 2013, 9, 977-994.

43 F. Santoro and D. Jacquemin, WIREs Comput. Mol. Sci., 2016, 6, 460-486.

44 D. Presti, F. Labat, A. Pedone, M. J. Frisch, H. P. Hratchian, I. Ciofini, M. C. Menziani and C. Adamo, J. Chem. Theory Comput., 2014, 10, 5577-5585. 
45 D. Presti, F. Labat, A. Pedone, M. J. Frisch, H. P. Hratchian, I. Ciofini, M. Cristina Menziani and C. Adamo, J. Comput. Chem., 2016, 37, 861-870.

46 D. Marx, ChemPhysChem, 2006, 7, 1848-1870.

47 O. K. Abou-Zied, R. Jimenez, E. H. Z. Thompson, D. P. Millar and F. E. Romesberg, J. Phys. Chem. A, 2002, 106, 3665-3672.
48 N. Kungwan, F. Plasser, A. J. A. Aquino, M. Barbatti, P. Wolschann and H. Lischka, Phys. Chem. Chem. Phys, 2012, 14, 9016-9025.

49 K. Benelhadj, W. Muzuzu, J. Massue, P. Retailleau, A. Charaf-Eddin, A. D. Laurent, D. Jacquemin, G. Ulrich and R. Ziessel, Chem. - Eur. J., 2014, 20, 12843-12857. 